\title{
Study on Scientific Location Level Classification and Optimization Methods of Military Equipment Maintenance Support Sites Network
}

\author{
Gong-da Yan, Peng Dong, Wei Lu and Peng Yu \\ Dept. of Management Engineering and Equipment Economic, Naval Univ. of Engineering, Wuhan 430033, China
}

\begin{abstract}
The scientific location of military equipment maintenance support sites problems are concerned by scholars. We study how to solve the location level classify without participation of experts, by setting up Hopfield neural network module, designing concrete computational procedures, and finally illustrating though an application case. The study can provide simple and practical theories and methods' reference for our military equipment maintenance support sites activities.
\end{abstract}

Keywords-Military equipment; Maintenance support sites; Location level classify

\section{INTRODUCTION}

The level of military equipment maintenance support sites are influenced by many aspects of the indicators [1], because these indicators are both qualitative and quantitative, and in different time, the dominant positions are also different for the imparity of military situation, so the relation of maintenance support sites' level and indicators are nonlinear in a certain time period. The study on military equipment maintenance support location based on neural network provides a new scientific ideas for location selection problems, and solves the problems about how to carry out the location selection without experts. In the absence of experts evaluation of maintenance support sites of the practical work level to select location can take use of historical data, through Hopfield neural network training, stimulating experts thinking, evaluating level of military maintenance support sites, and finally providing the basis for choosing the good maintenance support sites.

\section{ANALYSIS OF THE SCIENTIFIC LOCATION LEVEL}

\section{ClassifiCATION BASED ON HOPFIELD NEURAL NETWORK}

\section{A. The Basis Theory Analysis of Hopfield Neural Network}

Neural networks have been applied in many areas and location problems is a typical kind of decision problem [2, 3]. Hopfield network is a fully connected model of neural network, using structure features and method of study different from stratum neural network, stimulating memory mechanism of the biological neural network, and obtain satisfactory results [4]. The network and the learning algorithm was first put forward by American physicist in 1982, so it's called Hopfield neural network.

Network structure. Binary Neural Network was first proposed by Hopfield, the output of the neuron only take value of 1 and -1 , so it was also called Discrete Hopfield Neural Network, DHNN is a feedback network which is a single layer and the output is binary. For binary neurons, it's computation formula is as follows:

$$
u_{j}=\sum w_{i j} y_{i}+x_{i}
$$

$x_{i}$ means external output, and there also have:

$$
\left\{\begin{array}{c}
y_{j}=1, u_{j} \geq \theta_{j} \\
y_{j}=-1, u_{j}<\theta_{j}
\end{array}\right.
$$

A DHNN network state is the collection of information about output neural network, it's n neurons network for an output layer, which the status is a n-dimensional vector when it's time t.

$$
Y(t)=\left[y_{1}(t), y_{2}(t), \ldots y_{n}(t)\right]^{T}
$$

Because $y_{i}(t)(i=1,2, \ldots, n)$ can take value of 1 or $-1, \mathrm{n}$ dimensional vector $Y(t)$ has $2^{n}$ kinds of state, namely the network has $2^{n}$ kinds of states. Using $y_{i}(t)$ to represent the first $\mathrm{j}$ neurons, namely node $\mathrm{j}$ is in the time $\mathrm{t}$ state, thus the node's next time $\mathrm{t}+1$ state can pray:

$$
\begin{gathered}
y_{i}(t+1)=f\left[u_{j}(t)\right]=\left\{\begin{array}{c}
1, u_{j}(t) \geq 0 \\
-1, u_{j}(t)<0
\end{array}\right. \\
u_{j}(t)=\sum_{i=1}^{n} w_{i j} y_{i}(t)+x_{j}-\theta_{j}
\end{gathered}
$$

If $w_{i j}$ equals to 0 when $\mathrm{i}=\mathrm{j}$, it's means that the output of a neuron will not feed back to it's input, then DHNN is called nofeedback neural network. If $w_{i j}$ is not equal to when $\mathrm{i}=\mathrm{j}$, it means that the output of a neural will feed back to it's input, then DHNN is called self-feedback neural network.

Network workings. Hopfield network operates according to dynamics methods, it's working process is the evolution of the state of the neurons, that is, evaluating according to the direction of the "energy" (Lyapunov function) reduction from the initial state, until reach to a steady state, this state is the output of the network. There have two mainly forms of Hopfield network' workings:

a) Serial (asynchronous) working. At any time t, only a certain neuron i changes in accordance with formula (4) and formula (5), and other neurons' states are not changed. 
b) Parallel (synchronous) working. At any time t, the state of part of neurons or whole neurons will change in the meantime.

Network stability. Network stability is defined as: if the network is stable at some point, the state will no longer change, says that the network is in steady state.

$$
v(t+\Delta t)=v(t) \quad \Delta t>0
$$

Can be seen from the structure of DHNN: it is a kind of multiple input and contains Kan value of binary nonlinear dynamic systems. In the dynamic system, balanced steady state can be understood as a system's some kind of energy function in the process of system movement, it's energy declining and at last in a minimum value. Coben and Crossberg have given the sufficient conditions about Hopfield network' stability, pointed out that if Hopfield network' weight matrix W is a symmetric matrix, and the diagonal element is 0 , the network is stable. That is, in the weight matrix $\mathrm{W}$, if:

$$
\left\{\begin{array}{c}
w_{i j}=0, i=j \\
w_{i j}=w_{j i}, i \neq j
\end{array}\right.
$$

\section{B. The Applicability Analysis of Hopfield Neural Network}

Military equipment maintenance support sites level are divided into base level maintenance support sites, relay level maintenance support sites and grass roots level maintenance support sites. In the location task, generally, only base level maintenance support sites and the more important relay level maintenance support sites have the condition of experts' participating and assisting decision-making. In other conditions, maintenance support sites' location only can be got by authority leadership and supervisor through experience.

But it will give us great convenience and scientific basis if using neural network to assist decision-making [5,6]. Using neural network to solve location problems, assuming that there has a complex function relation between the conditions of maintenance support sites and the decision-making of maintenance support sites, it can be got through on-the-spot investigation and research by experts. Then, through neural network, using experts' successful location history as the training data, then the function relation can be fitted. The training is actually through the theory formula of the neural network, carrying on the function' multiple fitting one by one, closing to the experts' location function obtained by calculation. As a result of the function fitting of the neural network can be trained for many times to get close to the primitive function, considering the progress of computer technology and computational speed, thus through the computer to design neural network, the error between the function we fit and the function of experts can be infinitely narrow. Therefore, neural network can help us to make decisions.

\section{The Location Requirement Analysis of Military Equipment Maintenance Support Sites}

All kinds of construction requirements generally include the following aspects:

Invisibility. Avoiding foreign investigation at ordinary times is to prevent leaks in the enemy spy operations; avoiding enemy attack in wartime is to ensure our military equipment to be repaired and got “regeneration”, thus can be put into battle again.

Defense. It should have a certain capability of defense for more important military equipment maintenance support sites, in order to reduce the damage of enemy attack and our military casualties, ensure support sites have a certain capability of maintenance support.

Land prices. With the development of economy, it has to take cost into account. Today, even the military land also appears the difficulties of land requisition, and our military spending is limited after all, it can't be squandered at will. Therefore, land prices should be taken into account when we site.

Environment. Military equipment maintenance support sites location should be avoided setting up in high temperature, damp or other bad environment as far as possible, so as not to speed up the equipment wear and tear. It should be avoided setting up in busy streets or poor folk custom villages and towns, lest appearing leaks or dispute of military and civilian.

Hydrology. Military equipment maintenance support sites should be set up in coastal, along the river or around the terminal for large equipment or imported equipment in need of water.

Transportation. Good transportation is the need of carriage at ordinary times, but good transportation is necessary for operational security or dangerous evacuation in wartime. Transportation should be taken into consideration when locating support sites.

Area. According to the requirement put forward by equipment support management and user department, organizing related experts to determine after system demonstration, ensuring the area of military equipment maintenance support sites can meet the function of maintenance support sites, and adequate facilities can be equipped with for military equipment maintenance support.

Energy. Military equipment maintenance support sites should be set up in the place with enough energy supply, especially for higher degree support sites, on account of some large maintenance support equipments have huge consumption, therefore, energy has to be taken into account.

\section{The AnAlysis of Model Building Based on HOPFIELD NEURAL NETWORK}

Based on the analysis above, equipment maintenance center location mainly considers eight factors: hidden, defense, land prices, environment, hydrology, transportation, area and energy. Under the condition of hypothesis above indicators, designing calculation steps as shown in figure 1. 


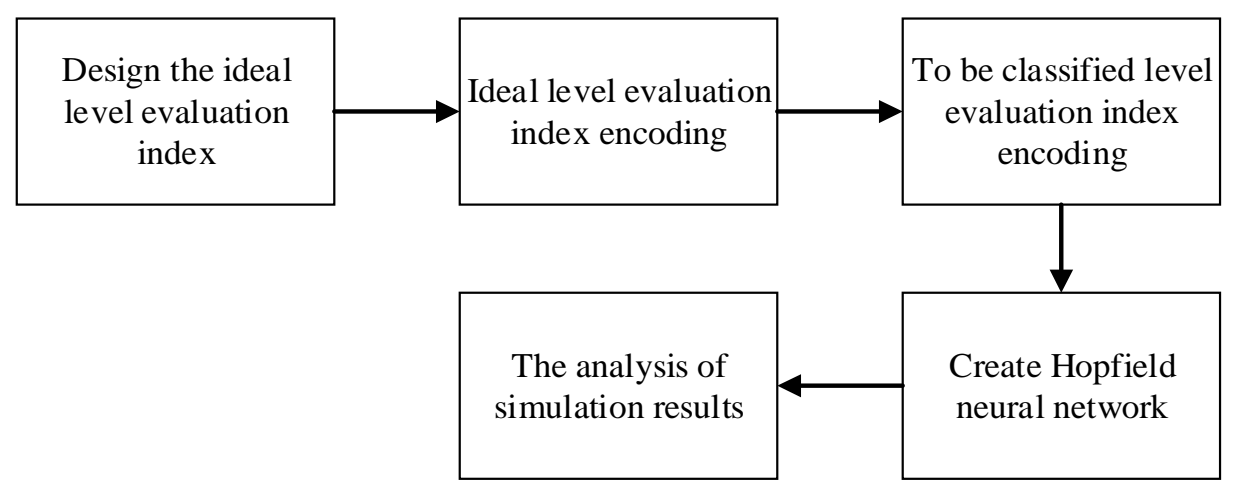

FIGURE I. THE FLOW CHART OF ESTABLISHED MODEL BASED ON HOPFIELD NEURAL NETWORK

Design the ideal level evaluation index. Assuming that there have ten historical schemes, fuzzy evaluation matrix is obtained by expert scoring method, if the scoring is on percentage system, to be normalized for table 1 .

Ideal level evaluation index encoding. The states of discrete Hopfield neural network neurons are only 1 or -1 , so it needs to be encoded when mapping evaluation index for the state of neurons. Coding rule is: when it is greater than or equal to the level of respect, the corresponding neurons state set to " 1 ", otherwise set to "-1".

Assuming that there have four evaluation schemes, history scheme is divided into four grades. Put each evaluation index average of each sample as the ideal evaluation index of each level. Namely, the balance of Hopfield neural network, such as the table 2. Corresponding four level evaluation indexes encoding of grade $\mathrm{A}, \mathrm{B}, \mathrm{C}, \mathrm{D}$.

To be classified level evaluation index encoding. Four scheme indexes to be evaluated shown as table3. The data of the scheme can be obtained by previous record query, can be obtained by professional field, and can also be obtained by the experience of authority department and supervisor, if the score is not between 0 and 1 , the data can be normalized between 0 and 1 , and code in the end, the coding result is shown in figure 3.

Create a network. After designing five ideal level evaluation indexes and encoding, using neural network toolbox built in MATLAB to create three type Hopfield neural network $[7,8]$.
Using the function newhop () of MATLAB neural network toolbox, the weight correction method the function using is the orthogonalization:

Step 1: input $\mathrm{N}$ input modes $t=\left\{t^{2}, t^{2}, \ldots, t^{N}\right\}$ and parameters $\tau, h$.

Step 2: calculate $A=\left\{t^{2}-t^{N}, t^{2}-t^{N}, \ldots, t^{N-1}-t^{N}\right\}$.

Step 3: do singular value decomposition for $\mathrm{A}$, and calculate the rank of $\mathrm{A} K=\operatorname{rank}(A)$.

Step 4: calculate $T^{P}=\sum_{i=1}^{K} u^{i}\left(u^{i}\right)^{T}$ and $T^{m}=$ $\sum_{i=K+1}^{K} u^{i}\left(u^{i}\right)^{T}$ by respectively using formula $U^{P}=$ $\left\{U^{1}, U^{2}, \ldots, U^{k}\right\}$ and $u^{m}=\left\{u^{K+1}, u^{K+2}, \ldots, u^{\mathrm{N}}\right\}$. $t^{N}$.

Step 5: calculate $W^{n}=T^{P}-\tau \times T^{m}$ and $b^{t}=t^{N}-W^{n} \times$

Step 6: calculate $\mathrm{W}=\exp \left(\mathrm{h} \times W^{t}\right)$.

Step 7: calculateb $=\mathrm{U} \times\left[\begin{array}{cc}C_{1} \times I(K) & 0(K, N-K) \\ 0(N-K, K) & C_{2} \times I(N-K)\end{array}\right] \times U^{T} \times b^{t}$,in which $C_{1}=\exp (h)-1$ and $C_{2}=-[\exp (-\tau \times h)-1] / \tau$.

Simulation and analysis. After creating a network, put the four to be classified scheme evaluation indexes as the input of Hopfield neural network, through the study of the agreed number, the policy results can be obtained. The simulation structure is as follows in figure 4 . From the results, plan 1 belongs to class $\mathrm{A}$, plan 2 belongs to class $\mathrm{B}$, plan 3 and plan 4 both belong to class $\mathrm{C}$. Thus the choice is plan 1 . 
TABLE I. GRADE EVALUATION INDEXES OF EACH SCHEME

\begin{tabular}{|c|c|c|c|c|c|c|c|c|c|}
\hline Scheme & Hidden & Defense & Land prices & Environment & Hydrology & Transportation & Area & Energy & Conclusion \\
\hline Scheme 1 & 1.00 & 1.00 & 1.00 & 1.00 & 1.00 & 1.00 & 1.00 & 1.00 & 1.00 \\
\hline Scheme 2 & 0.80 & 0.87 & 0.89 & 0.82 & 0.78 & 0.80 & 0.75 & 0.33 & 0.79 \\
\hline Scheme 3 & 0.67 & 0.93 & 0.22 & 0.75 & 1.00 & 0.80 & 0.49 & 0.66 & 0.74 \\
\hline Scheme 4 & 0.92 & 0.72 & 0.67 & 0.66 & 0.56 & 0.80 & 0.75 & 0.69 & 0.91 \\
\hline Scheme 5 & 0.87 & 0.93 & 1.00 & 1.00 & 1.00 & 1.00 & 1.00 & 1.00 & 0.96 \\
\hline Scheme 6 & 0.80 & 0.72 & 0.89 & 0.82 & 0.89 & 0.80 & 0.75 & 1.00 & 0.83 \\
\hline Scheme 7 & 0.67 & 0.72 & 0.67 & 0.66 & 0.67 & 0.60 & 0.49 & 0.66 & 0.69 \\
\hline Scheme 8 & 0.72 & 0.80 & 0.78 & 0.75 & 0.78 & 0.80 & 0.75 & 0.66 & 0.75 \\
\hline Scheme 9 & 0.60 & 0.60 & 0.56 & 0.58 & 0.56 & 0.60 & 0.49 & 0.66 & 0.58 \\
\hline Scheme10 & 0.47 & 0.47 & 0.44 & 0.41 & 0.44 & 0.40 & 0.49 & 0.35 & 0.51 \\
\hline
\end{tabular}

TABLE II. IDEAL FOUR LEVEL EVALUATION INDEXES

\begin{tabular}{|l|l|l|l|l|l|l|l|}
\hline $\begin{array}{l}\text { Index } \\
\text { Level }\end{array}$ & Hidden & Defense & Land prices & Environment & Hydrology & Transportation & Area \\
\hline A & 0.8780 & 0.8480 & 0.8900 & 0.8600 & 0.8460 & 0.8800 & 0.8040 \\
\hline B & 0.5480 & 0.7960 & 0.5580 & 0.7320 & 0.7800 & 0.6800 & 0.8500 \\
\hline C & 0.2820 & 0.2940 & 0.2860 & 0.2440 & 0.2380 & 0.2640 & 0.4920 \\
\hline D & $<0.2820$ & $<0.2940$ & $<0.2860$ & $<0.2440$ & $<0.2380$ & $<0.2640$ & 0.2640 \\
\hline
\end{tabular}

TABLE III. FOUR SCHEMES TO BE CLASSIFIED LEVEL EVALUATION INDEXES

\begin{tabular}{|c|c|c|c|c|c|c|c|c|c|}
\hline Scheme & Hidden & Defense & $\begin{array}{l}\text { Land } \\
\text { prices }\end{array}$ & Environment & Hydrology & Transportation & Area & Energy & conclusion \\
\hline Scheme1 & 0.9200 & 0.8000 & 0.8900 & 0.9200 & 0.8900 & 0.8000 & 1.0000 & 1.0000 & 1 \\
\hline Scheme 2 & 0.5200 & 0.8000 & 0.6700 & 0.8200 & 0.7800 & 0.6000 & 0.4900 & 0.7200 & 1 \\
\hline Scheme 3 & 0.6700 & 0.5200 & 0.4900 & 0.3200 & 0.4900 & 0.4000 & 0.1900 & 0.2900 & 1 \\
\hline Scheme 4 & 0.0800 & 0.2300 & 0.4600 & 0.3200 & 0.2900 & 0.3000 & 0.5400 & 0.3300 & 1 \\
\hline
\end{tabular}

$A$

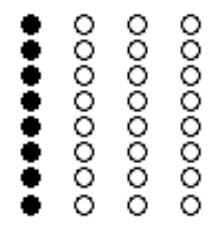

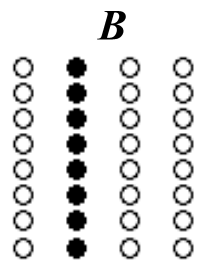

$C$

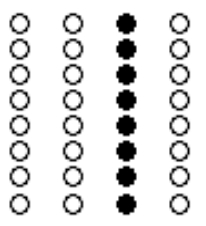

D

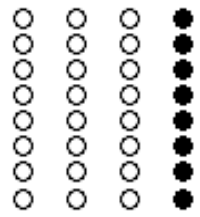

FIGURE II. IDEAL FOUR LEVEL EVALUATION INDEXES ENCODING
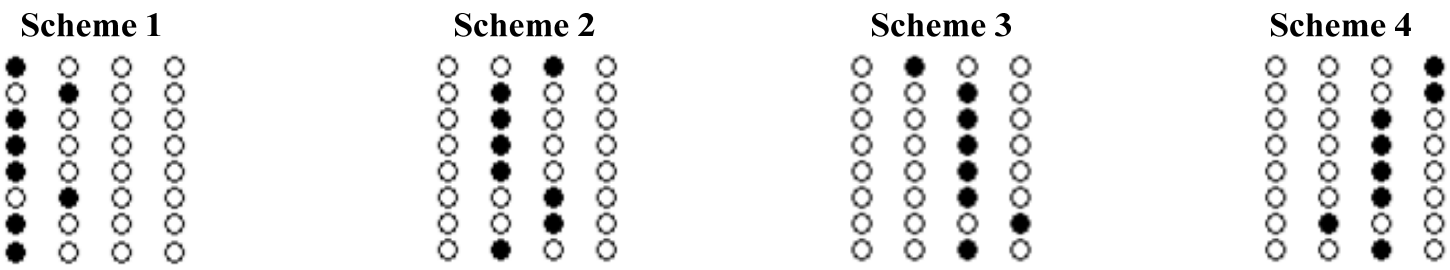

FIGURE III. FOUR SCHEMES TO BE CLASSIFIED LEVEL EVALUATION INDEXES ENCODING 


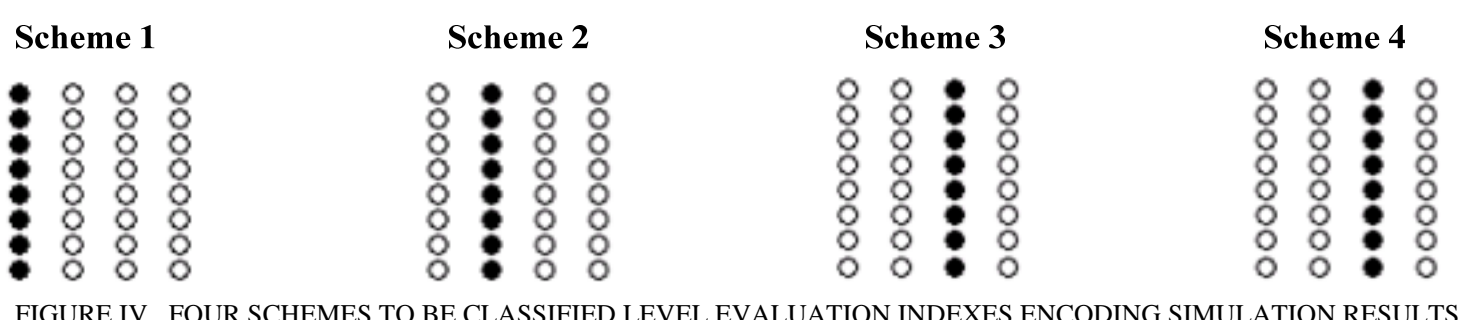

\section{IV.CONCLUSION}

This paper has mainly studied level classification problems of military equipment maintenance support sites. The location model aims to combine neural network with military maintenance support sites, so that the location task can be completed better and more quickly, especially at the basic level of equipment maintenance support sites in the absence of experts. Therefore, it has great important realistic significance and economic interests of the military for accelerating the formation of weapons and equipment's fighting effectiveness and security capabilities.

\section{ACKNOWLEDGEMENT}

This work is supported by Naval Engineering University Research Funding Project under Grant 20161613 and 20161614.

\section{REFERANCES}

[1] HUANG Yi-jia. A few questions need to be focused on our military equipment maintenance support power constriction under the condition of informatization. J. Equipment, 2007, 1(3): 2-3.

[2] HE Yu-bing, LI Xin-zhong. Neural network control technology and application. M. Science Press, 2000.

[3] WANG Xu, WANG Hong, WANG Wen-hui. Artificial neural network theory and application. M. Shenyang: Northeastern University Press, 2000.

[4] LI Guo-hui. The enterprise competitiveness evaluation model based on Hopfield neural network. J. Computer Science, 2011, 38(10): 1-2.

[5] WANG Wei. Artificial neural network theory-introduction and application. M. Beijing: Beijing University of Aeronautics and Astronautics Press, 1995.

[6] F. Fnaiech, D. Bastard, V Buzenac, R.Settineri, and M.Najim. A fast Kalman filter based new algorithm for training feedforward neural networks. J.Proc. EUSIPCO 94, Edinburg, U.K, 1994, 9: 13-16.

[7] GE Zhe-xue. Neural network theory and MATLAB R2007 implementation. M. Beijing: Electronic Industry Press, 2007.

[8] SHI Feng, WANG Xiao-chuan, YU Lei. Thirty cases analysis of MATLAB neural network. M. Beijing: Beijing University of Aeronautics and Astronautics Press, 2002. 